\title{
数控成型磨齿机加工误差在线监测及补偿*
}

\author{
赵飞 1 梅雪松 $^{1,2}$ 李光东 $^{1}$ 陶 涛 ${ }^{1}$ 姜歌东 ${ }^{1,2}$ \\ (1. 西安交通大学机械工程学院 西安 710049; \\ 2. 西安交通大学机械制造系统工程国家重点实验室 西安 710049)
}

\begin{abstract}
摘要: 研究数控机床加工过程在线监测及加工误差分析与补偿方法的问题, 提出用于数控成型磨齿机齿向误差在线监测及补 偿方法。数控机床加工过程中, 加工工件和刀具间的相对位置关系对机床加工精度具有重要影响。通过获取数控机床内置信 号(光柱、编码器)可以得到这一重要信息。基于内置信号同步采集方法, 利用齐次坐标变换原理对数控机床进给轴的内置信 号进行变换分析, 获得机床空间加工轨迹, 从而实现机床加工误差的在线监测与评估。同时, 通过分析加工过程中各进给轴 的动态位置信息, 可以确定影响加工误差的主要因素。基于分析结果, 通过软件实时补偿原理, 结合数控机床控制系统特点, 对主要误差源进行在线补偿, 从而达到提高加工精度的目的。采用该方法对一数控成型磨齿机加工过程齿向误差进行评估与 补偿, 与三坐标测量机检测结果对比表明该方法可以有效地获取加工误差形貌。根据基于分析结果进行补偿后, 使该机床齿 向误差明显降低, 提高了机床的加工精度等级。
\end{abstract}

关键词: 加工过程监测 精度测试 误差补偿 齐次坐标变换

中图分类号: TG659

\section{Machining Error Online Monitoring and Compensation of Numerical Control Forming Gear Grinding Machine}

\author{
ZHAO Fei ${ }^{1}$ MEI Xuesong ${ }^{1,2}$ LI Guangdong ${ }^{1}$ TAO Tao ${ }^{1}$ JIANG Gedong ${ }^{1,2}$
}

(1. College of Mechanical Engineering, Xi'an Jiaotong University, Xi'an 710049;

2. State Key Laboratory for Manufacturing Systems Engineering, Xi'an Jiaotong University, Xi'an 710049)

\begin{abstract}
The problem of machining error online monitoring and compensation method is studied, and the machining error online monitoring and compensation method is proposed which takes numerical control forming gear grinding machine as the research object. The relative position of work piece and tool is important for machining precision during machining process. One can get the relative position by testing the built-in signal which include encoder and direct encoder signal. In the proposed method, the space trace of machining is calculated from the built-in signal which is processed by the homogeneous coordinate transformation. The main factors that bring out the machining error can be determined by analyzing the processed built-in signal, and compensated into the numerical control system by online real time method. The proposed method is implemented on a numerical control forming gear grinding machine to improve its precision. Compared with the coordinate measuring machine test result, the proposed method can detect the form of the machining error. After compensating, the tooth alignment machining error is lowered obviously.
\end{abstract}

Key words : Machining process monitoring Precision test Error compensation Homogeneous coordinate transformation

\section{0 前言}

精度和效率是现代制造业最重要的因素之一, 因此大力发展高速高精度数控机床是世界各国制造

* 国家重点基础研究发展计划资助项目(973 计划, 2011CB706805)。 20120328 收到初稿, 20120914 收到修改稿
业的努力目标。高速高精度加工数控机床是机械系 统特性与控制系统特性相互耦合的复杂系统，其特 性不仅受几何误差和热变形误差的影响, 更受控制 系统参数匹配, 装配质量等引起的动态特性的影响。 对于高速、高精度的数控机床而言, 机床自身的几 何精度已经达到相当高的水平, 此时, 动态误差对 机床误差的影响更加明显, 甚至会成为影响机床加 
工精度的主要因素。因此, 研究数控机床动态特性 测试分析方法, 对提高数控机床加工精度具有重要 作用。

目前对数控机床进给系统动态特性测试的手 段主要有球杆仪 ${ }^{[1]}$ 、平面光梛 ${ }^{[2]}$ 等。HUA 等 $^{[3]}$ 提出 了一种新型的带有编码器的球杆仪, 提高了精度, 降低了成本, 增大了测量范围。球杆仪、平面光栅 等仪器可以对机床进给系统在空运行条件下的伺服 匹配特性、摩擦特性、换向间隙等因素进行分析。 上述方法虽然能准确地对数控机床几何误差和部分 动态特性进行测试, 却无法在机床切削过程中对机 床特性进行评估。因此, 研究者开始探索机床加工 过程监测方法。VOLKER 等 ${ }^{[4]}$ 利用编码器信号作为 动态特性测试信号, 实现了动态特性快速辨识。利 用这种所谓的内置传感器测试技术, 通过对比新旧 机床电流信号的变化特征, 实现了机床工况演变过 程监测。同时, 该文指出了随磨损的加剧, 进给系 统电流的变化特征。ERKORKMAZ 等 ${ }^{[5]}$ 利用无传感 器信息对进给系统丝杜及导轨的磨损、破损等故障 因素进行识别, 获取了良好的效果。同时该文指出 了传统测试方法在数控机床状态监测中的局限性和 无传感器测试的不足。YOUNG 等 ${ }^{[6]}$ 利用主轴电动 机电流对切削过程中切削力的变化进行了监测, 建 立了电动机电流信号与切削力变化的关系, 较准确 地对切削力进行预测。 $\mathrm{TONG}$ 等 ${ }^{[7]}$ 基于信息融合技 术通过结合刀具坐标信息对主轴运行过程中的电流 信息对加工过程进行监测, 实现了加工过程监测。 STEVEN 等 ${ }^{[8]}$ 对近十多年的数控机床加工过程监测 方法进行了回顾, 提出了对加工过程进行监测将促 进生产力的进步, 改善工件加工质量, 降低成本, 放宽对机床零部件设计加工的限制的观点。这些研 究大都集中在数控机床空切动态特性的检测与评估 方面, 数控机床加工过程监测方法还缺乏深入的 研究。

数控机床加工精度无法满足要求时, 一方面通 常需要对机床参数进行调整, 另一方面可以采取误 差补偿的方法。对这些加工过程误差进行补偿的方 法主要有软件补偿法和硬件补偿法两种 ${ }^{[9]}$ 。硬件误 差补偿的误差补偿量取决于补偿器, 而与机床成形 运动的实际误差无关, 且补偿量固定, 是一种静态 补偿方式。软件误差补偿可以实现误差的动态补偿, 根据实际误差修正误差补偿量。因此软件误差补偿 在理论上更有利于提高机床成形运动精度。目前对 误差的补偿的研究大多数还仅限于进给系统几何误 差如直线度、螺距误差、换向间隙等和摩擦特性、 热变形特性的补偿。杨建国 ${ }^{[10]}$ 建立数控车削中心热
分布模型, 利用 $\mathrm{A} / \mathrm{D}$ 电路操作控制器参数实现了热 误差的实时补偿。刘焕劳 ${ }^{[11]}$ 开发了基于一维步距规 的机床误差检测、精度评价和误差补偿系统实现了 华中数控系统的反向间隙补偿, 利用人工神经网络 模型对运动误差进行补偿。

本文基于理论分析与实践经验，提出了利用内 置传感器信息, 基于齐次坐标变换的数控机床加工 过程在线评估及补偿方法, 为实现数控机床加工精 度在线评估补偿提供了手段。文章内容安排如下: 信号获取分析及误差补偿原理在第 1 节进行了阐 述; 加工试验实施方案在第 2 节进行了叙述; 误差 特征及形成原因在第 3 节进行了分析, 同时对误差 补偿实施结果进行了对比; 最后在第 4 节中给出了 本文的结论。

\section{1 信号采集分析及补偿原理}

\section{1 信号分析方法}

一般在开放式数控系统中可以方便的获取三 类信号, 速度反馈信号、位置反馈信号及电流反馈 信号。为了在数控机床正常工作情况下进行数据采 集, 速度反馈信号、位置反馈信号采集时需要采用 三通采样方式; 电流信号可以通过伺服驱动器提供 的电流监测端口直接进行采集。速度反馈信号、位 置反馈信号的信号格式一般为三极管-三极管逻辑 (Transistor-transistor logic, TTL)方波、1Vpp 正弦、 Endat 等格式; 电流信号通常为 $\pm 10 \mathrm{~V}$ 的模拟量信 号。利用数控机床进给系统多种内置信号进行状态 监测, 需同时进行多种格式信号采样。本文通过统 一时基方法，利用板载时钟实现了多种格式信号的 同步采样, 并开发了相应的测试仪器, 为利用内置 信号进行数控机床加工状态监测提供了物质基础。 本文内置信号同步采样原理如图 1 所示。

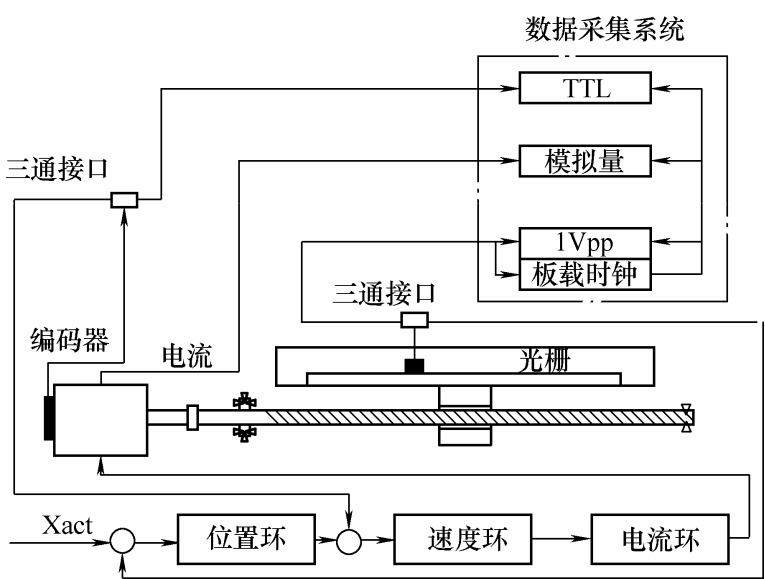

图 1 内置信号同步获取原理

考虑目前机床反馈环节中常用的信号格式, 图1 
中, 采用 $1 \mathrm{Vpp}$ 和 TTL格式信号计数卡采集位置及速 度反馈信号, 高速模拟量采集卡采集模拟量信号。 其中, 模拟量和TTL采集卡工作于从卡状态, 其工 作时钟由 $1 \mathrm{Vpp}$ 计数卡提供。1 Vpp 计数卡通过主通道 时钟向其他卡提供工作时钟, 并控制同步采集过程。 三通接口根据驱动器相应接口的定义进行转接, 并 与采集设备连接。通过上述方法可以在不干涉机床 运行状态的情况下同步获得机床不同类型的信号。

通过内置信号分析数控机床加工状态, 需要对 内置信号信息与进给系统动力学特性的相互关系进 行映射, 从而获取信号特征与数控机床加工状态间 的关系。进给系统内置信号 (电流、编码器、光栅等) 与进给系统动力学特性的关系分别陈述如下。

(1) 电流信号。当伺服电动机驱动滚珠丝杜和 工作台运动的时, 伺服电动机电流和负载间存在如 下关系

$$
T=K_{t} i_{q}=J \ddot{\theta}+\tau_{a}+\tau_{f}
$$

式中, $T$ 为电动机输出转矩; $K_{t}$ 为电流转换系数; $J$ 为系统转动惯量; $\theta$ 为伺服电动机转角; $\tau_{\alpha}$ 为扰 动力矩; $\tau_{f}$ 为摩擦力矩。当伺服电动机驱动滚珠丝 杜匀速运动时, 转动惯量 $J \ddot{\theta}=0$, 因此式(1)演变为

$$
i_{q}=\frac{\tau_{a}+\tau_{f}}{K_{t}}
$$

由式(2)可知, 通过进给轴的电流信号可以对由 于扰动和摩擦引起的动态特性进行监测。

(2) 位置和速度反馈信号。由于全闭环数控机 床各轴的位置及速度传感器相对独立, 因此轴间的 相对几何关系被假设为理想状态, 通过内置信号无 法反映轴间的几何精度特性。然而, 同一轴上的位 置和速度传感器的信号包含工作台与电动机间相对 运动的动态过程, 其包含着进给轴运动过程中由进 给系统安装、几何精度、润滑条件等引起的进给系 统动态特性。分析位置和速度传感器信号可以获取 进给系统运动过程中的工作台位置波动情况, 实现 运动过程的精度监测评估。需要指出的是, 采集进 给系统内置信号, 不影响数控机床加工过程。因此, 采用内置信号对数控机床加工过程进行监测评估具 有较高的可行性。速度反馈信号 $\theta_{e n}$ 和位置反馈信号 $d_{s l}$ 的理论关系为 $k \theta_{e n}=d_{s l}$ 。其中, $k=p / i ; p$ 为 丝杜导程; $i$ 为减速机构减速比。受机械及控制特 性的影响, 速度反馈信号 $\theta_{e n}$ 和位置反馈信号 $d_{s l}$ 间 不可避免的存在偏差

$$
E=k \theta_{e n}-d_{s l}
$$

偏差 $E$ 主要由零部件的制造误差, 零部件受力 产生的弹性变形及系统传动过程中的动态特性等造
成的误差构成, 如式(4)所示

$$
E=\Delta_{c}+\Delta_{d}+\Delta_{g}
$$

式中, $\Delta_{c}$ 为系统动态特性误差, $\Delta_{d}$ 为弹性变形引 起的偏差, $\Delta_{g}$ 为零部件制造误差引起的偏差。

由式(4)可知, 偏差 $E$ 表明, 工作台的位置和伺 服电动机位置在传动过程中受各因素的影响并不能 保持一致。通过计算进给系统位置和速度传感器的 反馈信号间的偏差 $E$, 可以获得数控机床加工过程 中工作台位置相对于伺服电动机位置的动态位置波 动。该精度不影响进给系统静态的定位精度, 但在 加工过程中, 动态位置波动将会影响工件的加工质 量。在数控机床加工过程中, 受切削力等因素的影 响, 偏差 $E$ 中的动态特性误差, 弹性变形误差等将 被放大。加工过程中工作台的位置和伺服电动机位 置的相对变化过程信息在偏差 $E$ 中得以反映。因 此, 通过分析偏差 $E$ 中包含的信息特征, 可以实现 进给系统加工过程加工精度的监测与评估。

\section{2 空间误差模型}

五轴联动数控机床一般包含三个直线轴及两 个旋转轴, 通过五轴联动基本可以完成各种复杂曲 面的加工。在加工过程中由于几何误差、热变形误 差和动态误差等因素的影响, 使理论轨迹和实际轨 迹间存在偏差，该偏差将直接复印至被加工工件形 面上, 影响加工精度。如第 1.1 节所述, 通过计算 参与于运动的进给轴的运动偏差 $E$, 可以对上述误 差进行评估。通过齐次坐标变换, 将各运动轴的运 动过程中的偏差耦合计算, 得到最终复映至加工表 面的误差量。为了达到通过监测机床插补轴的位置 反馈信号和速度反馈信号来监测加工形状误差的目 的，工件与刀具的相对运动需变换到绝对坐标系下 进行描述。本文假设绝对坐标系标架(Absolute coordinate system, ACS) 与机床床身固连, $X_{A} 、 Y_{A}$ 和 $A_{A}$ 的方向分别与机床坐标系(Machine coordinate system, MCS)的 $X_{M}, Y_{M}$ 和 $Z_{M}$ 轴相同。坐标变换如下 所示

$$
\left(\begin{array}{c}
{ }^{A} \boldsymbol{P} \\
1
\end{array}\right)=\left(\begin{array}{cc}
{ }^{A} \boldsymbol{R}_{B} & { }^{A} \boldsymbol{P}_{B} \\
0 & 1
\end{array}\right)\left(\begin{array}{c}
{ }^{B} \boldsymbol{P} \\
1
\end{array}\right)
$$

式中, ${ }^{B} \boldsymbol{P}\left(X_{M}, Y_{M}, Z_{M}\right)$ 为点在机床坐标系下的位 置, ${ }^{A} \boldsymbol{P}\left(X_{A}, Y_{A}, A_{A}\right)$ 为点在绝对坐标系下的位置。 ${ }^{A} \boldsymbol{R}_{B}$ 和 ${ }^{A} \boldsymbol{P}_{B}$ 分别为坐标旋转及坐标平移系数矩阵。 分别将速度传感器信号和位置传感器信号变换至 ACS 下, 然后计算偏差 $E$ 在各个方向的分量, 可以 得到工件和刀具在相对运动过程中理论轨迹与实际 轨迹间的偏差。为显示偏差 $E$ 在进给过程中的波动 情况, 将偏差 $E$ 在主进给方向展开, 则可以得到偏 
差 $E$ 依位置变化的情况。以主进给方向为 $Z$ (运动坐 标为 ${ }^{B} \boldsymbol{P}_{Z}$ ) 的情况为例, 进给轴位置波动造成的工件 加工误差在 $X 、 Y$ 方向的误差如式(6)所示

$$
\left(\begin{array}{c}
{ }^{A} \boldsymbol{P}_{x} \\
{ }^{A} \boldsymbol{P}_{y} \\
{ }^{A} \boldsymbol{P}_{z} \\
1
\end{array}\right)=\left(\begin{array}{cccc}
r_{11} & r_{12} & r_{13} & \delta_{1} \\
r_{21} & r_{22} & r_{23} & \delta_{2} \\
r_{31} & r_{32} & r_{33} & \delta_{3} \\
& 0 & & 1
\end{array}\right)\left(\begin{array}{c}
{ }^{B} \boldsymbol{P}_{d x} \\
{ }^{B} \boldsymbol{P}_{d y} \\
{ }^{B} \boldsymbol{P}_{Z} \\
1
\end{array}\right)
$$

式中, ${ }^{A} \boldsymbol{P}_{x},{ }^{A} \boldsymbol{P}_{y},{ }^{A} \boldsymbol{P}_{z}$ 加工点在绝对坐标系下的坐标 值, ${ }^{B} \boldsymbol{P}_{d x}$ 和 ${ }^{B} \boldsymbol{P}_{d y}$ 为在机床坐标系下的相对误差, $r_{i j}$ 为坐标旋转变换系数, $\delta_{i}$ 坐标偏移变换系数。

\section{3 误差补偿策略}

对加工过程实现误差动态补偿, 需要对控制数 控机床轴插补的指令位置进行实时修正。施耐德公 司的 NUM 开放式数控系统的动态操作功能为实现 上述功能提供了可能。所谓动态操作, 即指在数控 系统每个扫描周期内持续执行的一种操作, 直到系 统得到取消该操作的指令, 它可以为插补指令叠加 一个附加值。该操作周期为 $6 \mu \mathrm{s}$ 。因此, 基于本文 所提出的加工误差在线监测方法, 对数控机床参与 运动的轴误差特性进行测试分析, 通过动态操作方 式, 对其误差值进行补偿。补偿原理: 首先检测绘 制参与运动的轴的全行程误差曲线; 根据误差曲线 拐点及误差曲线形状, 对造成误差超差的主要拐点 进行反向加权补偿值计算, 最后利用动态操作功能 将反向加权补偿计算值实时补偿到机床轴运动中。

\section{2 在线监测及补偿试验}

YK73200 数控成型砂轮磨齿机床具有 $X Y Z A C$ 等五个联动轴, 和一个用于砂轮修型的 $W$ 轴。其中, $X$ 轴和 $Z$ 轴与机床床身固定, $A$ 轴安装于 $Z$ 轴工作 台上, $Y$ 轴叠加在 $A$ 轴工作台上, 通过 $A$ 轴的旋转 实现斜齿轮的加工; $C$ 轴安装于 $X$ 轴上, 如图 2a 所示。 $Z$ 轴和 $Y$ 轴光栅每毫米刻线数均为 50 刻线, $C$ 轴编码器线数为 36000 线, $A$ 轴编码器线数为 18000 线。控制器采用 NUM1050 数控系统。在该 数控磨齿机试切调试过程中, 出现异常误差, 机床 精度无法达到设计精度。为提高该机床加工精度, 对其磨削过程进行监测分析。

测试过程中本文利用三通分线盒直接从驱动 器反馈接口获取信号。采集过程中, 同步采集该机 床 $Y$ 光栅、 $Y$ 编码器、 $Z$ 光栅、 $A$ 光栅、 $C$ 光栅等 5 通道信号, 采样频率 $1 \mathrm{kHz}$ 。加工过程和空切过程 的各轴动态位置信号利用课题组自行开发的测试仪 器(图 2b)进行采集。

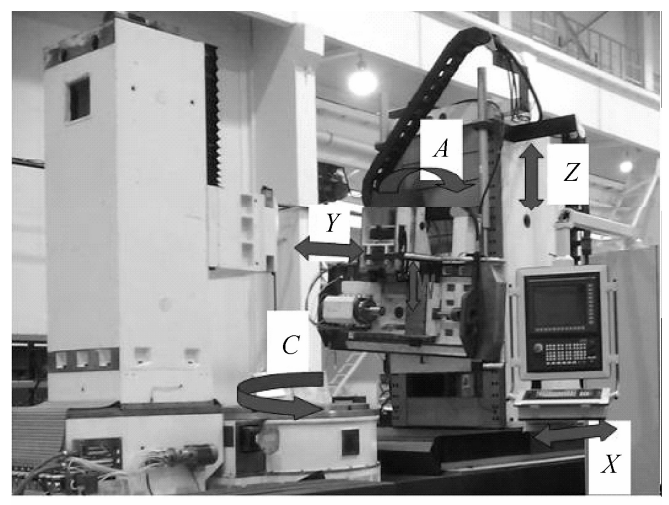

(a) 测试机床结构

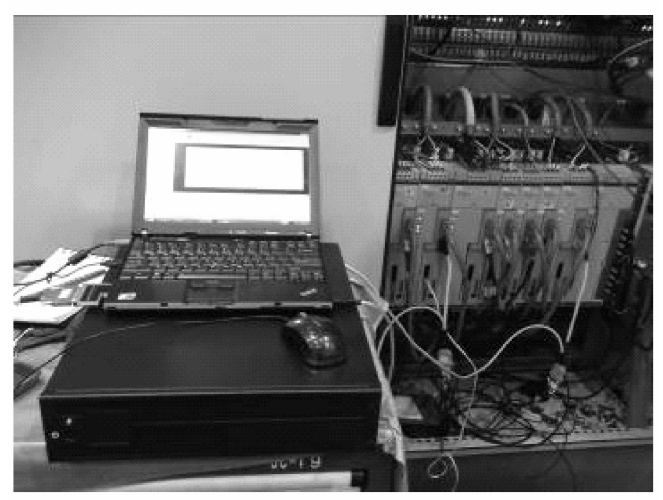

(b) 测试现场

图 2 测试机床与测试仪

监测过程中, 被切削工件为 60 齿, 模数 25 , 齿厚 $200 \mathrm{~mm}$ 的直齿轮。轮齿磨削过程如下: $C$ 轴转 动使被磨削齿槽处于既定位置, $Z$ 轴垂直方向进给 实现齿槽方向的切削, 在此过程中 $X, Y, A$ 和 $C$ 轴 始终保持理论锁止的状态。每个齿 $Z$ 轴齿槽方向进给 4 次, 2 次粗磨, 2 次精磨。砂轮最大直径为 $400 \mathrm{~mm}$ 。 监测时, 设定 $Z$ 轴的进给速度为 $2400 \mathrm{~mm} / \mathrm{min}$ 。

切削过程中各被监测轴的动态位置如图 3 所 示。 $A$ 轴信号在空切过程中出现幅值微小的与 $Z$ 轴 运动相关的位置漂移; 在加工过程中, $A$ 轴的位置 漂移幅值未产生较大变化, 但在 $8 \mathrm{~s}$ 区出现异常波 动; $C$ 轴位置信号在正反向空切过程中出现近似对 称的位置波动, 波动幅值较小, 但在加工过程中, 该波动的对称性出现变化, 幅值没有明显改变; $Y$ 轴位置信号在空切过程中波动趋势与 $C$ 轴类似, 且 幅值微小。但在加工过程中, $Y$ 轴位置信号幅值增 大近 4 倍, 正负向加工过程中, 位置波动的一致性 加强。通过对比空切和加工过程可知, 受切削过程 刀具和工件相互作用的影响, 被监测各轴的位置信 号出现了明显的变化。为提高该台数控机床的加工 精度, 需要对加工过程数据进行进一步分析, 获得 影响加工精度的主要因素, 并进行有效的补偿。监 测过程对多个齿槽加工过程进行了监测, 各个齿的 加工过程具有很高的一致性, 因此图 3 中仅给出了 
一个往复加工过程的数据图形。
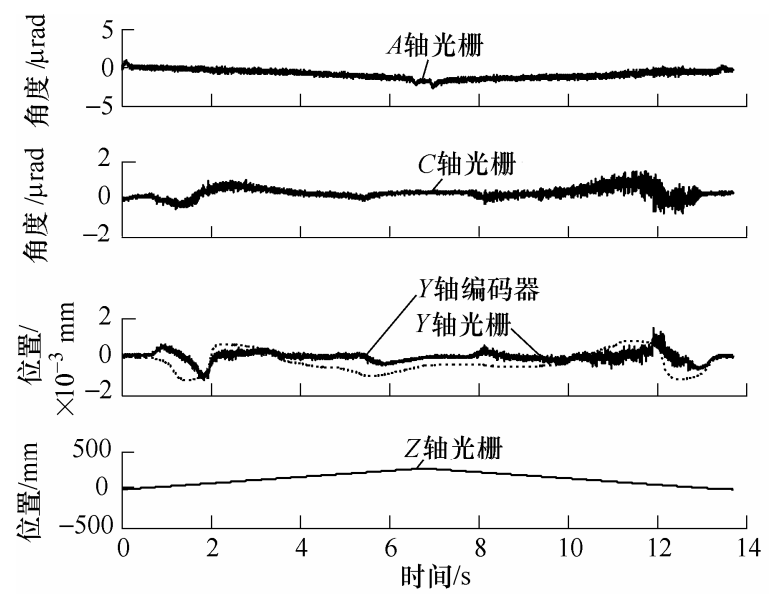

(a) 空切测试下内置传感器信号
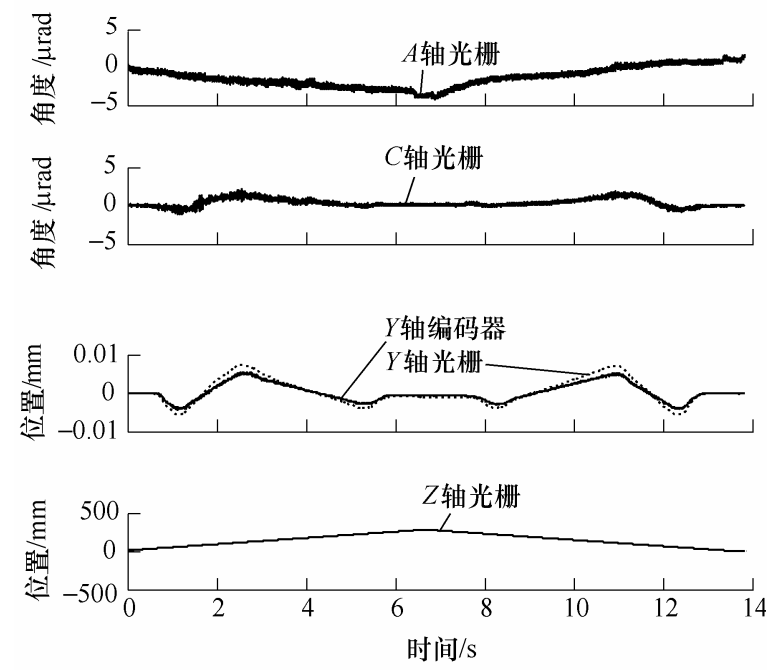

(b) 切削条件下内置传感器信号

图 3 空切和切削条件下内置传感器信号对比

\section{3 监测特性分析及补偿}

\section{1 加工过程特性分析}

为了分析各轴的轨迹偏差对空间路径的影响, 利用第 1.2 节所提出的方法, 对数据进行处理。设 绝对坐标系原点固结于被加工齿轮圆心, $X$ 方向与 机床 $X 、 Y 、 Z$ 轴的方向相同, 方向遵循右手法则。 刀具坐标系相对于绝对坐标系 ACS 沿机床 $X$ 轴负 方向偏移 $-750 \mathrm{~mm}$, 刀具坐标系 $B$ 相对于绝对坐标 系 $A$ 绕 $X$ 轴旋转, 旋转角度由 $A$ 轴光栅进行测量, 然后刀具坐标系 $B$ 再绕绝对坐标系 $A$ 的 $Z$ 向旋转, 旋转角度由 $C$ 轴光棶测量。依据式(6)和被测机床结 构, 参与运动的各轴位置波动造成的加工误差在绝 对坐标下各坐标轴上的投影依据式(7)计算。式中, ${ }^{A} \boldsymbol{P}_{x},{ }^{A} \boldsymbol{P}_{y},{ }^{A} \boldsymbol{P}_{z}$ 为齿轮齿向各点在绝对坐标系下的坐 标值, ${ }^{B} Y_{e r}, A_{e r}, C_{e r}$ 为数控机床参与运动的轴的速度 传感器和位置传感器测量值机床坐标系下的偏差,
${ }^{B} Z_{s l}$ 为 $Z$ 轴的运动位置

$$
\begin{aligned}
& \left(\begin{array}{c}
{ }^{A} \boldsymbol{P}_{x} \\
{ }^{A} \boldsymbol{P}_{y} \\
{ }^{A} \boldsymbol{P}_{z} \\
1
\end{array}\right)=\left(\begin{array}{cccc}
\cos C_{e r} & -\sin C_{e r} & 0 & 0 \\
\sin C_{e r} & \cos C_{e r} & 0 & 0 \\
0 & 0 & 1 & 0 \\
0 & 0 & & 1
\end{array}\right) \times \\
& \left(\begin{array}{cccc}
1 & 0 & 0 & -750 \\
0 & \cos A_{e r} & -\sin A_{e r} & 0 \\
0 & \sin A_{e r} & \cos A_{e r} & 0 \\
0 & & 1
\end{array}\right)\left(\begin{array}{c}
0 \\
{ }^{B} Y_{e r} \\
{ }^{B} Z_{s l} \\
1
\end{array}\right)
\end{aligned}
$$

通过坐标转换分析后, 空切及加工过程中切削 刀具切削点相对于绝对坐标系的运动状态如图 4 所 示。对比空切运动过程和切削过程, 齿轮磨削条件 和空切运行条件下由测试数据所得到的轮齿的齿向 误差曲线趋势基本一致, 曲线的主要拐点坐标处于 $Z$ 轴相近的位置。空切过程中, 正负向往复测试重 复性较差, 在 $200 \sim 300 \mathrm{~mm}$ 区间差别较大; 加工过 程中, 正负向往复测试的一致性较好, 仅在 $100 \mathrm{~mm}$ 附近在 $X$ 向出现一定的偏差。空切和加工过程中的 幅值差别较大, 且形状也出现变化。空切过程误差 具有随机的波动, 加工过程中误差仅具有 2 个明显 的拐点。为区别两个行程, 在图 $4 \mathrm{~b}$ 中将正行程误差 曲线沿 $X$ 向偏置 $1 \times 10^{-4} \mathrm{~mm}$ 。

为分析偏差的幅值, 将其分别投影至 $O X Z$ 和 $O Y Z$ 平面进行分析。投影后的结果如图 5 所示。加 工过程中空间误差趋势主要分布在 $Y$ 方向, 分布在 $X$ 方向上的很小，这是由于齿向误差对 $X$ 方向的误 差不敏感，而 $Y$ 方向的误差则直接复映到齿向误差 上。空间误差曲线有 3 个拐点, 可以分为 4 部分进 行分析。在 $Z$ 轴方向 20 43 mm 内, 空间误差向 $Y$ 轴正向增加, 在 43 $80 \mathrm{~mm}$ 内, 空间误差曲线向 $Y$ 轴负向增加, 并越过零点, 且达到正向误差最大值。 在 80 209 mm 内, 空间误差再次向正向增加。在 最后的一个阶段, 空间误差又一次换向。切削条件 下, 空间误差在 $Y$ 方向的最大值为 $0.0087 \mathrm{~mm}$, 在 $X$ 方向的最大值为 $10^{-4} \mathrm{~mm}$ 。空切条件下, $Y$ 向的最 大误差值为 $0.0013 \mathrm{~mm}, X$ 向的最大误差值为 $10^{-9} \mathrm{~mm}$ 。切削条件下误差幅值约为空切条件的 8 倍。将切削条件下的空间误差解耦到参与运动的轴 $X$ 和轴 $Y$ 上, 如图 5 所示, $X$ 向误差分量极微小, 可以忽略不计, 而 $Y$ 轴误差分量波动达到 $8.7 \mu \mathrm{m}$, 显然 $Y$ 向所引起的误差为齿向加工误差的主要误差 源。 $Y$ 向误差方向与机床的 $Y$ 轴相同, 因此 $Y$ 向误 差主要由被测机床的 $Y$ 轴引起。为区别两个行程, 在图 $5 \mathrm{~b}$ 中将正行程误差曲线沿 $X$ 向偏置 $-1 \times 10^{-4} \mathrm{~mm}$ 。 


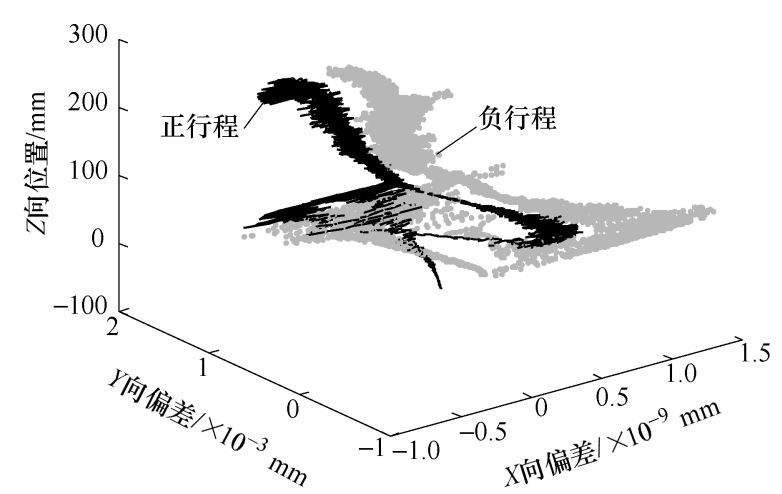

(a) 空切条件下空间误差图谱

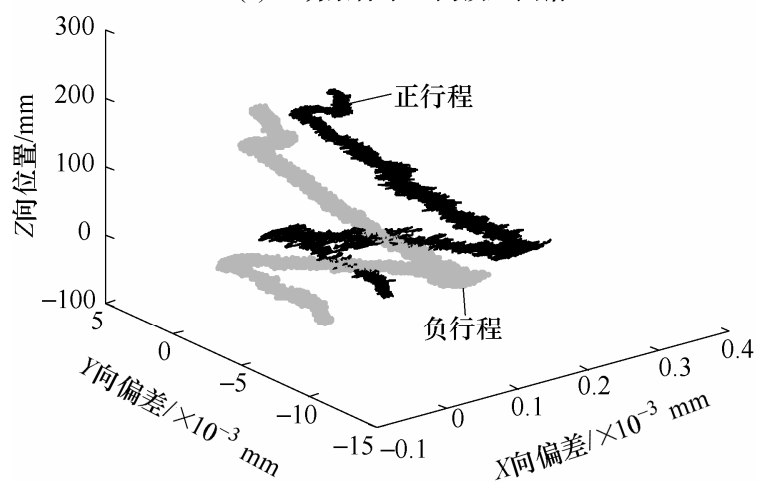

(b) 加工条件下空间误差图谱

图 4 加工和空切下空间误差图谱

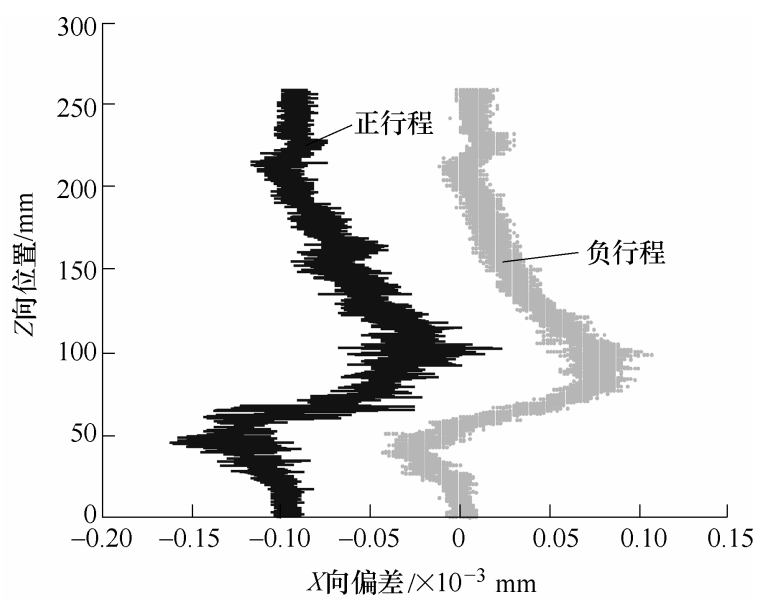

(a) $X$ 轴误差解耦

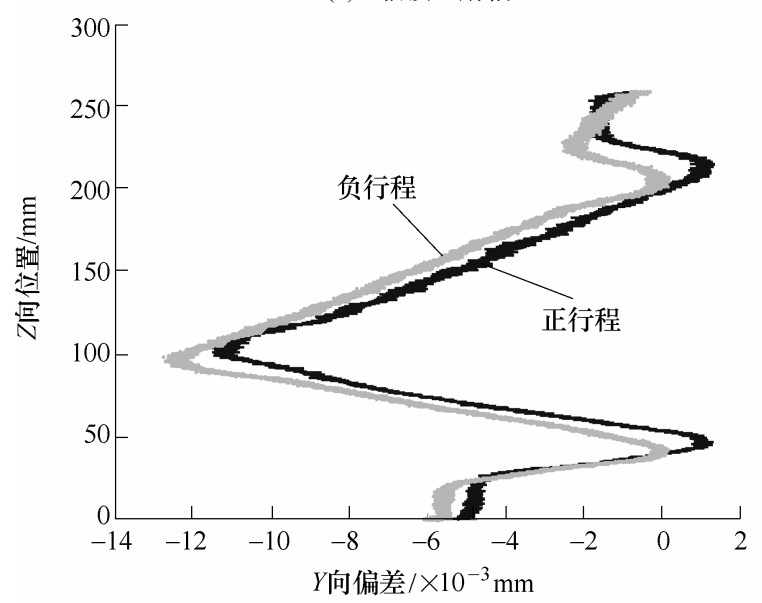

(b) $Y$ 轴误差解耦

图 5 误差主因素分析
为对本文所提误差检测方法进行对比，磨削后 的齿轮被送到检测部门利用三坐标测量机进行鉴 定。测试报告结果扫描图如图 6a 所示。图 6a 中, 共测量 3 个齿槽, 左侧为齿槽左侧的三个齿面的齿 向误差测量结果，相应的右侧为相同齿槽的右齿面 齿向误差测量结果。横向每一格为 $10 \mu \mathrm{m}$, 纵向每 一格为 $40 \mathrm{~mm}$ 。三个齿槽的测量曲线几乎相同, 均 在 $60.6 \mathrm{~mm}$ 附近产生较大的误差, 齿槽左面和右面曲 线形状相似。齿槽轮齿切削齿向误差最大值为 14.2 $\mu \mathrm{m}$, 最小值为 $5.3 \mu \mathrm{m}$ 。对比本文所提方法的空间误 差曲线形状与测试报告曲线形状可知, 两种测试结 果形貌基本一致。由于本文所提方法未考虑切削变 形及机床进给系统几何误差的影响, 因此, 在幅值 上必然存在一定的误差。

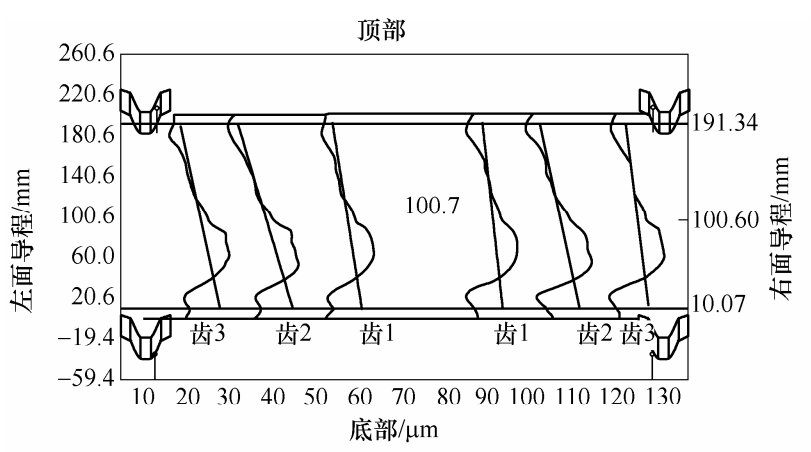

(a) 补偿前

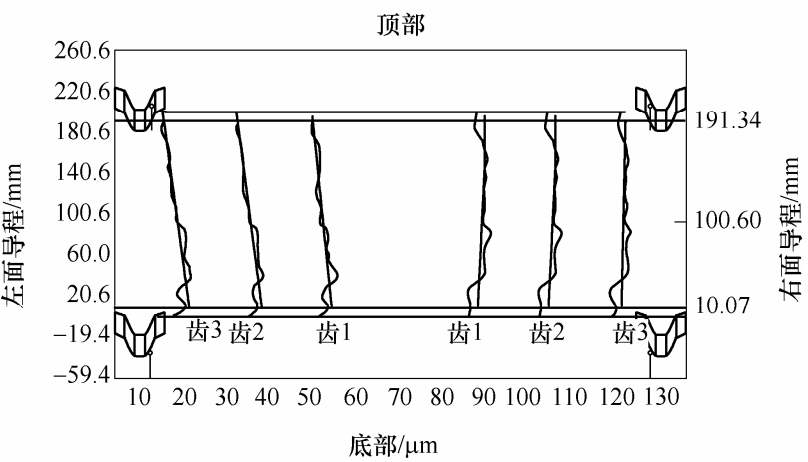

(b) 补偿后

图 6 三坐标测量机测量结果

\section{2 误差补偿}

在得到齿向误差空间误差曲线后，分析得到齿 向误差主要由 $Y$ 轴进给系统异常造成, 且该误差具 有很强的重复性, 在多次加工后仍存在。因此采用 NUM 数控系统提供的动态操作方法对加工过程指 令位置进行干预。对空间误差在 $O Y Z$ 平面的投影, 分别确定 3 个拐点值, 对拐点间误差进行反向补偿。 补偿后, 对同一齿轮进行磨削。磨削后的齿轮测量 报告扫描图如图 6b 所示, 其比例与图 6a 相同。通 过测试报告对比可以发现，在影响齿向误差最大的 区域, 误差幅值有明显的变化, 左齿面最大误差为 $6.3 \mu \mathrm{m}$, 右齿面最大误差为 $5.4 \mu \mathrm{m}$, 均小于 GB 2001 
公差要求。通过对比分析可知, 经补偿后数控磨齿 机齿向加工误差达到机床设计要求。

\section{4 结论}

(1) 基于内置传感器信号同步采集方法, 实现 了数控成型磨齿机齿向误差的在线监测评估, 并利 用评估结果对数控机床加工误差进行实时补偿。实 践证明本方法快速、有效。

(2) 监测过程中, 利用齐次坐标变换及误差解 耦方法对同步采集的内置传感器信息进行处理, 获 取齿向误差空间图谱及轴向误差图谱。与坐标测量 机测试结果对比, 本文所提出的监测方法可以较准 确的实现齿向加工误差的在线监测与评估。本方法 可扩展至其他类型数控机床。

（3）基于误差在线监测评估结果和软件实时补 偿原理, 利用 NUM 系统所提供的动态操作方式对 机床精度进行补偿, 有效地提高了试验对象的精度。

(4) 本文方法的试验验证对象为采用 NUM 数 控系统的数控成型磨齿机, 对于其他数控系统及机 床类型的加工误差补偿方法还需要进一步研究。

\section{参 考 文 献}

[1] KAKINO Y, IHARA Y, NAKATSU Y. The measurement of motion error of $\mathrm{NC}$ machine tools and diagnosis of their originals by using telescoping magnetic ball-bar method[J]. Annals of the CIRP, 1987, 36(1): 377-380.

[2] DU Zhengchun, ZHANG Shujie, HONG Maisheng. Development of a multi-step measuring method for motion accuracy of NC machine tools based on cross grid encoder[J]. International Journal of Machine Tools and Manufacture, 2010, 50(3): 270-280.

[3] HUA Qiu, LI Yan, LI Yanbin. A new method and device for motion accuracy measurement of NC machine tools, part 1: Principle and equipment[J]. International Journal of Machine Tools and Manufacture, 2001, 41(4): 521-534.

[4] VOLKER P, MANFRED W. Sensorless machine tool condition monitoring based on open $\mathrm{NCs}[\mathrm{C}] / /$ Proceedings of the 2001 IEEE International Conference on Robotics \& Automation, 2001, 3(3): 3104-3108.
[5] ERKORKMAZ K, WONG W. Rapid identification technique for virtual $\mathrm{CNC}$ drives[J]. International Journal of Machine Tools and Manufacture, 2007, 47: 1381-1392.

[6] YOUNG H J, DONG W C. Estimating cutting force from rotating and stationary feed motor currents on a milling machine $[\mathrm{J}]$. International Journal of Machine Tools and Manufacture, 2002, 42(14): 1559-1566.

[7] TONG Liang, YAN Ping, LIU Fei. Monitoring computer numerical control machining progress based on information fusion[J]. Chinese Journal of Mechanical Engineering, 2012, 24(6): 1074-1081.

[8] STEVEN Y L, ROGELIO L H, ROBERT G L. Machining process monitoring and control : The state-of-the-art[J]. Journal of Manufacturing Science and Engineering, 2004, 126(2): 297-310.

[9] 李晓丽. 面向多体系统的五轴联动数控机床运动建模 及几何误差分析研究[D]. 成都: 西南交通大学, 2008.

LI Xiaoli. Multi-body system-oriented kinematics modeling and geometric error analysis for five-axis NC machine tools[D]. Chengdu : Southwest Jiaotong University, 2008.

[10] 杨建国. 数控机床误差综合补偿技术及应用 $[\mathrm{D}]$. 上 海: 上海交通大学, 1998 .

YANG Jianguo. Error synthetic compensation technique and application for $\mathrm{NC}$ machine tool[D]. Shanghai: Shanghai Jiao Tong University, 1998.

[11] 刘焕劳. 数控机床几何误差测量及误差补偿技术的研 究[D]. 武汉：华中科技大学, 2005 .

LIU Huanlao. Research on the Geometric error measurement and error compensation of the numerical control machine tools[D]. Wuhan: Huazhong University of Science and Technology, 2005.

作者简介: 赵飞, 男, 1983 年出生, 博士研究生。主要研究方向为数控 机床动态特性测试分析、信号处理和故障诊断。

E-mail: zhaofayei@yahoo.cn

梅雪松(通信作者), 男, 1963 年出生, 教授, 博士研究生导师。主要研 究方向为运动控制与数控系统、激光加工和精密测试技术。

E-mail: xsmei@mail.xjtu.edu.cn 